\title{
Comment concilier économie et écologie dans l'estuaire de la Loire
}

\author{
par M. Leroy \\ Délégué Général d'ACEL \\ (Association Communautaire de l'Estuaire de la Loire)
}

Le plan national de relance de 1976 prévoyait l'installation d'un terminal méthanier dans l'estuaire, qui complétait la vocation énergétique de cet ensemble industrialo-portuaire, à côté de deux centrales électriques, de la raffinerie de Donges et d'un projet de terminal charbonnier. Un débat complexe avait divisé les partisans du site de l'estuaire de la Seine et ceux du site de l'estuaire de la Loire... Mais l'installation d'un terminal méthanier à l'embouchure de la Loire avait l'avantage d'entraîner un programme d'ensemble dont allait bénéficier le Port de Nantes/St-Nazaire et la raffinerie de Donges. Cet avantage réel d'aménagement du territoire fit arbitrer en faveur de la Loire.

\section{I $⿴ 囗$ LE PROGRAMME DE 1976 - 1980}

Depuis le début du siècle les ingénieurs du Port s'efforçaient de faciliter l'entrée de la marée dans l'estuaire afin de permettre la remontée des bateaux jusqu'à Nantes, et ceci selon deux principes :

- la constitution d'un important bassin de marée à l'amont de Nantes,

- la régularisation du chenal de la mer à Nantes à la fois en "plan» (redressement, confortation des berges et suppression des îles) et en "coupe» (suppression des seuils rocheux et approfondissement progressif vers l'aval), bref une «mise en trompette" de l'estuaire.

Or le creusement d'un chenal méthanier devait être l'occasion -à travers de nombreuses péripéties- de parfaire l'embouchure de la trompette et de supprimer le hiatus que constituaient la Passe des Brillantes et les Vasières de Lavau. Plus précisément, il permettait de creuser le chenal aval, entre Donges et la mer, de - $9 \mathrm{~m}$ à - 13,25 m, ce qui devait, la suite l'a montré, assurer le fonctionnement de la raffinerie pendant de très longues années et maintenant encore.

L'évidente logique économique de cette démarche et les imperfections des premières réflexions d'environnement se conjuguèrent pour étouffer toute autre préoccupation : les aménagements de l'estuaire qui furent décidés en 1980 ne purent prendre aucune précaution importante d'environnement, ni dans l'information, ni dans la réalisation ; l'affaire du «bras de Lavau» en fut une illustration caricaturale. Pourtant le Préfet de Région avait pris une décision originale pour l'époque et courageuse : étendre l'obligation d'étude d'impact à l'ensemble de l'estuaire, sous forme d'une "étude générale d'environnement» comportant un état 0 avant travaux.
Le programme d'aménagement décidé comprenait donc le creusement du chenal méthanier, le remblaiement de plusieurs zones portuaires au nord et au sud de l'estuaire (Lavau et Carnet) et la constitution d'un îlot-digue au milieu de la Loire : le banc de Bilho, amorçant le côté sud de la mise en trompette.

Dans cette tranche d'aménagement de l'estuaire, de 1976 à 1980 , on peut donc considérer que les conceptions «brutalistes" de choix ont prévalu, l'environnement étant considéré comme un alibi suspect freinant le progrès économique, voire trahissant l'intérêt national. Pourtant l'attitude de l'Etat (étude générale d'environnement) indiquait une ouverture que, par la suite, le Conseil scientifique des études d'environnement de l'estuaire de la Loire (C.S.E.E.L.) allait confirmer, du moins formellement. En effet -et c'est le propos de cette communication- il faut reconnaître que la suite de l'aménagement de l'estuaire (1991-1996) a été conduite de façon beaucoup plus nuancée, plus ouverte et plus soucieuse des enjeux majeurs d'environnement, c'est ainsi que l'on peut parler d'une méthode de choix d'aménagements plus «réformiste».

\section{VINGT ANS PLUS TARD : LA SITUATION EN 1996}

Comme en 1976, il s'agit de continuer la mise en trompette de l'estuaire couplée avec le remblaiement de zones portuaires.

Précisément le programme "pris en considération» par le ministre de l'Equipement en 1991 comprend :

- le recreusement du chenal de Donges Est (ex «Lavau»), - la constitution d'une digue au milieu de la Loire (dite «virgule de Paimboeuf»),

- le remblaiement définitif de la zone de Donges Est et sa consolidation.

Si ce programme est beaucoup moins coûteux que 20 ans auparavant, il est inspiré par la même doctrine d'aménagement estuarien (la mise en trompette) et il en renforce les effets. La virgule de Paimboeuf étant la suite du Banc de Bilho.

Dans ces conditions où est la différence ? et où se manifeste une conception plus «réformiste» ?

Sachant qu'aucune décision n'est prise, la différence réside à ce jour dans la méthode d'étude conduisant au choix. 


\section{III 圆 UNE EVOLUTION REFORMISTE}

- La première nouveauté est la participation des collectivités territoriales organisées, avec le Port, au sein de l'Association Communautaire de l'Estuaire de la Loire. C'est ainsi qu'ACEL' ${ }^{1}$ a pris les initiatives suivantes :

- de confier (en 1992) un audit à un expert indépendant mais très qualifié (Rapport $\mathrm{F}$. Essig $\mathrm{N}^{\circ} 1$ ) qui a préconisé :

- un aménagement mesuré et à moyen terme d'une réserve foncière portuaire,

- précédé d'une évaluation hydraulique de l'ensemble de l'estuaire effectuée par un organisme indépendant des protagonistes du programme de 1976-1980,

- de faire faire (en 1993) cette évaluation par le Laboratoire de Delft. Les conclusions de cette évaluation ont été acceptées par l'ensemble des protagonistes, à savoir : tester les ouvrages projetés sur un modèle mathématique.

- La deuxième nouveauté semble résider dans l'attitude de l'Etat qui apparaît arbitrer, davantage que par le passé, entre les enjeux portuaires et ceux des équilibres environnementaux, et ceci à travers plusieurs réactions ou plusieurs décisions :

- son attention aux audits de F. Essig et du Laboratoire de Delft et ceci malgré ses prérogatives régaliennes en matière d'eau,

- sa participation financière et technique importante à la modélisation mathématique,

- le Plan Loire qui a rappelé l'objectif national de relèvement de la ligne d'eau, la nécessité d'une modélisation ainsi que la compensation nécessaire à tout aménagement aval,

- sa proposition d'un «Programme Concerté» d'aménagement, de développement et de protection de l'estuaire de la Loire, auquel il a associé les travaux de l'ACEL et les conclusions d'un expert qu'il a désigné sur l'estuaire.

Cette évolution de la méthode prend, on le voit, un caractère quasi-institutionnel croissant, qui consacre le caractère réformiste de nos conceptions d'aménagement, tant de la part des collectivités que de l'Etat. Mais si l'évolution des structures est décisive, c'est plutôt celle des techniques qui doit nous intéresser ici. De ce point de vue, la modélisation mathématique, pilotée de façon partenariale par les services du Préfet de Région et l'ACEL, est certainement l'innovation la plus importante.

\footnotetext{
1 ACEL est une association réunissant à titre personnel

- Monsieur Luc DEJOIE - Président du Conseil Général de Loire Atlantique - Président d'ACEL

- Monsieur Olivier GUICHARD - Président du Conseil Régional

- Monsieur Jean-Marc AYRAULT - Député-Maire de Nantes

- Monsieur Joèl BATTEUX - Maire de St Nazaire

- Monsieur Yannick GUILLEMOT - Président de Port Atlantique

- Monsieur Alain MUSTIERE - Président de la C.C.I. de Nantes

- Monsieur Frank LANG - Président de la C.C.I. de St Nazaire

- Monsieur Didier LORIEUX - Président de l'Union Maritime de la Basse Loire
}

\section{IV 口 LA MODELISATION MATHEMATIQUE DE LA LOIRE ESTUARIENNE}

Malgré quelques réticences réflexes, le principe d'une modélisation mathématique d'ensemble a été retenu car l'objectif est d'éclairer les choix de politiques d'aménagement en testant éventuellement plusieurs propositions, voire en les combinant. Des modélisations plus spécifiques deviendront nécessaires plus tard, quand interviendront les problèmes de réalisation, sans doute posés par les services compétents de l'Etat.

La maîtrise d'ouvrage de l'opération ayant été confiée à l'Agence de l'Eau Loire Bretagne, c'est elle qui a conçu le cahier des charges en concertation avec les services de l'Etat, des collectivités d'ACEL et du Port. Des experts et des partenaires de l'enseignement supérieur ont aussi été consultés.

La modélisation est suivie par un groupe technique et un comité consultatif. Elle se déroule en trois phases :

- La première est une évaluation à dominante hydraulique réalisée par un modèle simple, associée à un bilan des études passées. Elle comporte notamment une simulation de l'évolution de l'estuaire sans aucun travaux et un premier test des projets identifiés aujourd'hui.

- La seconde phase, plus longue, s'appuiera sur des modèles plus élaborés ( $2 \mathrm{D}$ et $3 \mathrm{D}$ suivant les questions) elle affinera les hypothèses retenues après la première phase et accentuera l'analyse qualitative de l'eau et des milieux humides.

- La troisième phase -conditionnelle- associera les organismes d'enseignement et de recherche à la modélisation.

L'accent et la priorité mis sur l'hydraulique sont motivés par le fait que le facteur dominant de l'équilibre de l'estuaire est la présence de l'eau et son régime : en effet, les analyses les plus fines seraient radicalement remises en cause si le régime hydraulique, ou la hauteur de la ligne d'eau, devaient être modifiés.

L'équipe choisie pour la réalisation de cette modélisation de la Loire estuarienne est franco-danoise; c'est une association du BCEOM et du Danish Hydraulic Institute. Les raisons du choix ont été l'utilisation de méthodes simples et progressives (modèles de complexité croissante avec les exigences de l'analyse), le souci de la traduction en termes d'aménagement, l'indépendance et l'objectivité de l'équipe, et enfin, le respect du budget ( $8 \mathrm{MF}$ environ).

Ce budget étant alimenté par :

- le Ministère de l'équipement $20 \%$ et de l'environnement

- les collectivités territoriales d'ACEL .........................20\%

- l'Agence de l'Eau, le Port et .........................................20 \% les Voies Navigables de France

- l'Union européenne $40 \%$

et se répartit approximativement en :

$-50 \%$ pour la constitution d'une base de données complétant celle du Port Autonome

$-50 \%$ pour la modélisation proprement dite et les tests. 


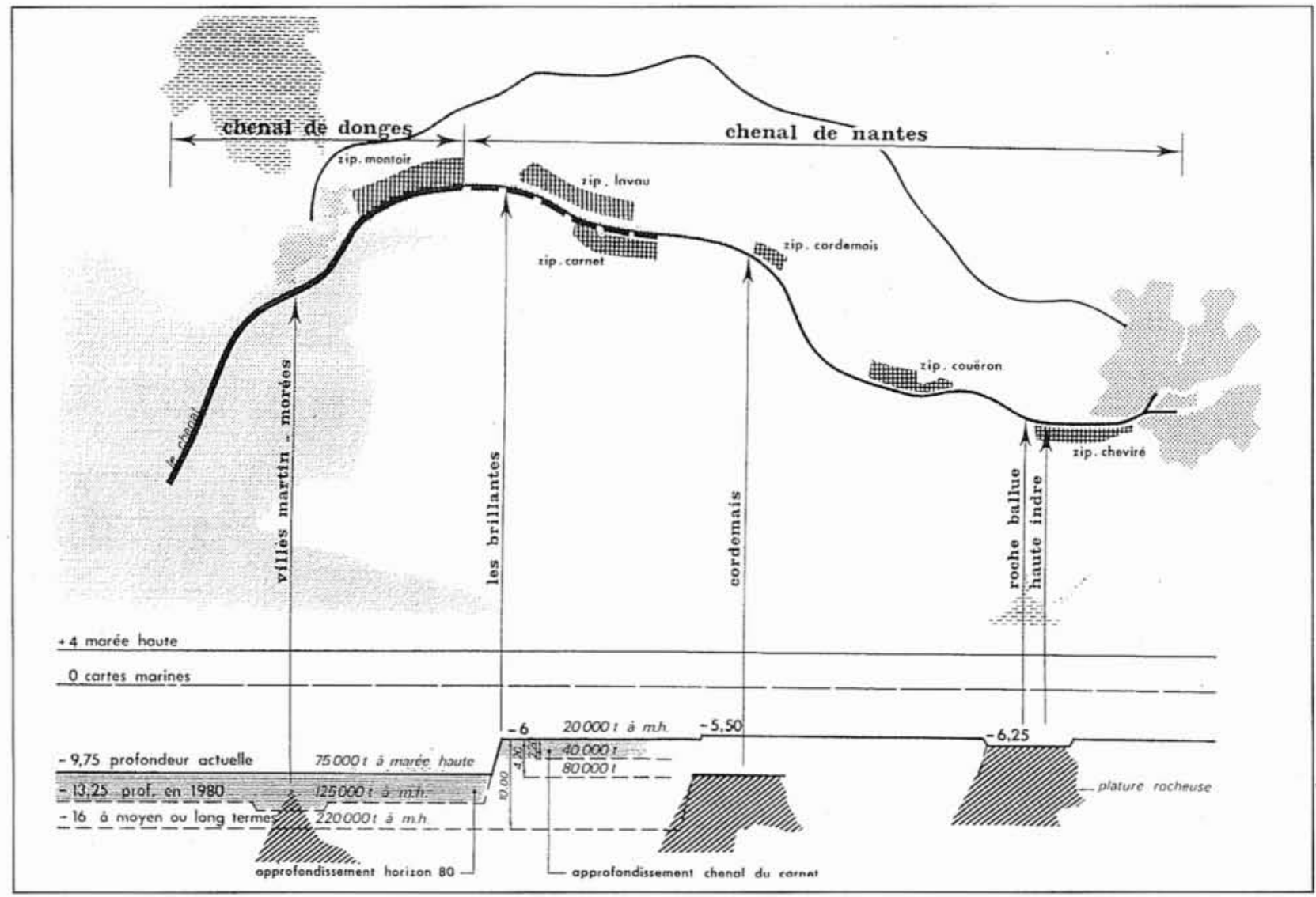

Coupe longitudinale sur le chenal maritime.

\section{DESULTATS PROVISOIRES OU TENDANCES DE LA REFLEXION?}

Il est important de rappeler que l'objectif principal de cette demande est :

- de rechercher une politique d'ensemble sur la Loire estuarienne plutôt qu'une juxtaposition de solutions techniques proposées par tronçons,

- d'apporter des solutions élaborées paritairement entre l'Etat et les collectivités d'une façon dont l'objectivité ne puisse pas être contestée.

Il n'est donc pas possible pour ACEL, avant les résultats des deux premières phases de la modélisation -et a fortiori avant même les résultats de la première phase- de proposer des solutions d'aménagements équilibrées pour l'ensemble de la Loire estuarienne. On peut, tout au plus, déceler des premières tendances :

- la responsabilité dominante des désordres de l'estuaire incombe au surcreusement du bassin de marée à l'amont de Nantes, ce qui doit confirmer définitivement les travaux du Port, du CSEEL et du Laboratoire de Delft...

- ... et rend plus aléatoire toute proposition de rééquilibrage de l'estuaire ou de remontée de la ligne d'eau fondée sur des aménagements exclusivement placés à l'aval.

Ainsi, aucune des solutions d'aménagement portuaire actuellement à l'étude ne peut améliorer, à elle seule, la situation. En revanche, aucune ne semble devoir aggraver directement cette situation, à l'exception de l'évolution de la Passe Sud pour laquelle on ne dispose d'aucune tendance chiffrée le 15 avril 96 . Enfin le rôle dominant du bassin de marée semble donner beaucoup d'intérêt à toute solution de remontée de la ligne d'eau située à proximité de l'agglomération nantaise, ainsi que l'avait déjà suggéré le «rapport Dạmbre».

Mais ne peut-on pas résumer les premières tendances de notre réflexion? Par exemple de la façon suivante :

- Nous disposons désormais d'un outil d'étude globale de politiques d'aménagement avec lequel nous pourrons tester, non seulement chaque aménagement proposé, mais aussi les recommandations du Plan Loire et les solutions les plus appropriées, notamment au relèvement de la ligne d'eau ou au bon fonctionnement de la Passe Sud...

- ... l'important programme d'aménagement prévu au contrat de plan doit être l'occasion d'amplifier ces recommandations, voire de les compléter, non seulement à l'aval mais encore plus à l'amont...

- ... et ceci dans le cadre d'une concertation qui doit rendre les solutions fiables et faire cesser les polémiques, quelquefois injustifiées, que nous avons pu connaître à propos de I'aménagement, et désormais du rééquilibrage, de la Loire estuarienne. 